\title{
Influence of non-glide stresses on plastic flow: from atomistic to continuum modeling
}

\author{
V. Vitek ${ }^{a}$, M. Mrovec ${ }^{a}$, J.L. Bassani ${ }^{b, *}$ \\ ${ }^{a}$ Department of Materials Science and Engineering, University of Pennsylvania, Philadelphia, PA 19104, USA \\ ${ }^{\mathrm{b}}$ Department of Mechanical Engineering and Applied Mechanics, University of Pennsylvania, Philadelphia, PA 19104, USA
}

\begin{abstract}
The Schmid law, which is accurate for face-centered-cubic (fcc) metals, assumes that only the shear stress acting in the slip plane in the slip direction controls the plastic deformation. Hence, it is implicitly assumed that the critical resolved shear stress (CRSS) for the slip is not affected by any other components of the applied stress tensor. This rule is almost ubiquitously utilized in large-scale continuum computations of plastically deforming single and polycrystals. On the other hand, in materials with more complex structures and for some orientations of the dislocation line the cores can spread onto several non-parallel planes. The most widely-known example is the screw dislocation in bcc metals, though this phenomenon is quite universal in structures that are not close packed. Signatures of such core configurations commonly include unexpected deformation modes and slip geometries, strong and unusual dependence of flow stresses on temperature and strain rate, a high Peierls stress, and, in general, a breakdown of the Schmid law. In this paper, we first summarize results of atomistic computer simulations of the response of $1 / 2\left\langle\begin{array}{lll}1 & 1 & 1\end{array}\right\rangle$ screw dislocations to the applied shear stresses. The calculations have been made using central-force many-body potentials and tight-binding based bond-order potentials for molybdenum. While the core structure found is not the same for the two descriptions of atomic interactions, both lead to a very similar orientation dependence of the critical resolved shear stress for the dislocation motion which takes place along the most highly stressed $\left\{\begin{array}{lll}1 & 1 & 0\end{array}\right\}$ plane. This dependence reveals the break down of the Schmid law invoked by the effect of shear stresses acting in another $\left\{\begin{array}{lll}1 & 1 & 0\end{array}\right\}$ plane, which are called non-glide stresses. These results are then transferred to macroscopic level by formulating single crystal yield criteria that include the effects of non-glide components of the stress tensor. These criteria form a basis for multislip yield criteria and flow relations for continuum analyses. Using this approach we demonstrate that the effects of non-glide stresses that have their origin at the level of individual dislocations also have significant effect on polycrystalline response, including a significant tension-compression asymmetry.
\end{abstract}

(C) 2003 Elsevier B.V. All rights reserved.

Keywords: Polycrystals; Multislip; Atomic interactions; Dislocation core structure; Yield criteria

\section{Introduction}

Increasingly, three-dimensional (3D) simulations involving large plastic deformations are being integrated into both the design process of metallic materials and the design and manufacturing of components that support a wide range of technologies. Examples include powder and deformation processing and components ranging from turbine blades to microelectronic packages to name a few. Both Lagrangian and Arbitrary Lagragian-Eulerian finite element implementations are the basis for commercial codes. However, the physical basis of the models, specifically the yield criteria

\footnotetext{
* Corresponding author. Tel.: +1-215-898-5632; fax: +1-215-573-7932.

E-mail address: bassani@seas.upenn.edu (J.L. Bassani).
}

that enter simulation codes for inelastic flow, are nearly always based on the Schmid law [1-4]. This law states that glide on a given slip system, defined by a slip plane and direction of slip, is controlled by the resolved shear stress on that system, the Schmid stress, and in a rate-independent formulation commences when this stress reaches a critical value [5] (see also [6]). Implicitly, other components of the stress tensor and, for well-annealed crystals, the sign of stress, play no role in the deformation process. Materials in which this law always applies possess close-packed structures, which primarily includes face-centered-cubic (fcc) and hexagonal close packed (hcp) materials when the glide is restricted to basal planes. The reason is dissociation of dislocations into Shockley partials that keeps them confined to the close-packed planes for every orientation of the dislocation line and, therefore, they possess planar core structures. 
On the other hand, in materials with more complex and open structures the cores may spread into several nonparallel planes for some orientations of the dislocation line. Such core structure may then very significantly affect the macroscopic plastic flow. Common signatures of such effects are: unexpected deformation modes and slip geometries; strong and unusual dependence of flow stresses on crystal orientation and temperature; and, most significantly, a break down of the Schmid law (for reviews, see [7-11]). A general reason for the break down of the Schmid law is the impact of the non-glide stresses upon the dislocation motion via their influence on the dislocation cores. These stresses are usually shear stresses acting either in the direction of the Burgers vector in a plane other than the slip plane, or perpendicular to the Burgers vector; the latter cannot induce dislocation motion but can alter the dislocation core. The most prominent example of materials in this category are body-centered-cubic (bcc) metals [8,9,12-15], but the class of such materials is much broader and includes intermetallic compounds $[7,9,10,16-18]$, oxides and semiconductors [11,19-24] and even organic and geological materials [25-27].

These non-planar atomic core configurations generally give rise to a unique stress-state dependence of flow properties that can significantly affect critical phenomena such as strain localization, evolution of the texture of grains, and failure mechanisms at mesoscopic and macroscopic length scales. An understanding of the stress-state dependence exists in the case of fcc metals [1-3,28,29] and some close-packed compounds [4,17,30,31], but essential information is usually lacking in models and simulations for non-close-packed materials. This information is the exact tensorial nature of the stress-state dependence of plastic flow that originates at the level of individual dislocations, which then projects into flow relations for realistic simulation of the plastic response of single crystals, polycrystals and, ultimately, components made from such materials.

In bcc transition metals the break down of the Schmid law arises from the non-planar spreading of the cores of $1 / 2\langle 111\rangle$ screw dislocations that is generally regarded as controlling many important features of slip in these materials $[8,9,12-15,32-34]$. Two distinct deviations from Schmid law can be identified. First, the critical resolved shear stress (CRSS) for slip may depend on the shear stress in the slip direction acting in different planes of the core spreading that could be alternative slip planes. Secondly, the CRSS may be influenced by other components of the applied stress tensor, in particular shear stresses in the direction perpendicular to the Burgers vector [34-38]. In the present paper, we focus only on the effect of shear stresses in the slip direction acting on planes into which the core spreads. In the case of the $1 / 2\langle 111\rangle$ screw dislocation these are three $\left\{\begin{array}{lll}1 & 10\end{array}\right\}$ planes of the $\left\langle\begin{array}{llll}1 & 1 & 1\rangle & \text { zone. }\end{array}\right.$

The dependence of the CRSS on the orientation of the maximum resolved shear stress plane (MRSSP) was studied by atomistic modeling of the glide of the $1 / 2\langle 111\rangle$ screw dislocation using several different methods for description of atomic interactions in transition metals. We concentrate here on molybdenum for which such study was made using central-force many-body potentials [38], potentials based on a modified generalized pseudopotential theory (MGPT) [39-41], screened bond-order potentials [42-45] as well as an ab initio pseudopotential plane-wave method within the local-density approximation of the density functional theory $[46,47]$. While the details of the core structure are not the same in these calculations the calculated dependence of the CRSS on the orientation of the MRSSP is very similar in all cases. This dependence demonstrates the breakdown of the Schmid law arising from the effect of shear stresses acting in the three $\left\{\begin{array}{lll}1 & 1 & 0\end{array}\right\}$ planes that contain the [1 111$]$ direction and comprises the well-known twinning-antitwinning asymmetry of slip observed in bcc metals (see, e.g. $[8,14])$.

Using a continuum framework for the effects of non-glide stresses $[30,31]$ that was employed earlier in the case of $\mathrm{Ni}_{3} \mathrm{Al}[4,17]$, we construct the yield criterion that can accurately reproduce the stress-state dependence found in the atomistic simulations for molybdenum. This criterion, which captures the slip characteristics arising from the atomic structure and properties of dislocation cores, is then employed in a Taylor-type calculation for uniaxial stressing of a random polycrystal of bcc grains with $12\left\{\begin{array}{lllll}1 & 1 & 0\end{array}\right\}\left\langle\begin{array}{llll}1 & 1 & 1\end{array}\right\rangle$ slip systems. This calculation demonstrates that a significant non-Schmid effect, including a tension-compression asymmetry, results at the polycrystal level. Moreover, earlier studies [4] have shown that hardening can further amplify the effects of non-glide stresses in polycrystals. These findings illustrate indubitably that the stress-state dependence of plastic flow, which originates at the level of individual dislocations, projects all the way to the plastic behavior of polycrystals. The understanding and characterization of these aspects of plastic behavior require multiscale modeling involving atomic level simulations on the scale of nanometers to provide input into continuum calculations on the scale from microns to meters.

\section{Atomistic simulations of screw dislocations in molybdenum}

Many experimental and theoretical studies performed in the last 40 years have established beyond doubt that the 1/2[1 111$]$ screw dislocations control the essential aspects of the plastic deformation of bcc metals, specifically the temperature and stress-state dependence of plastic flow (for reviews, see $[7-9,14,15,33])$. The most prominent characteristic of these dislocations is their core which spreads into several non-parallel planes of the [1 111 l] zone, as first recognized by Hirsch [32], and confirmed by many atomistic studies that all find the core spreading into three $\left\{\begin{array}{lll}1 & 10\end{array}\right.$ planes intersecting along the [1 111$]$ direction. Earlier calculations, which employed pair-potentials to describe atomic 
interactions, always found that the core is asymmetrically spread into three $\{110\}$ planes, similarly as in Fig. 1a; [48-50]. Since this core structure is not invariant with respect to the $\left[\begin{array}{ll}10 & \overline{1}\end{array}\right]$ diad, a symmetry operation of the bcc lattice, another energetically equivalent configuration exists in which the spreading of the core into three $\{110\}$ planes is found on the other side relative to the line of their intersection. This core structure is, therefore, called degenerate. However, in recent calculations both the degenerate core and the non-degenerate core, which is spread symmetrically into the three $\left\{\begin{array}{lll}1 & 1 & 0\end{array}\right\}$ planes of the [1 111 l] zone, were found. An example of the latter is shown in Fig. 1b. Which of these two types of the core is found depends on the description of interatomic forces and even when two alternate descriptions represent the same material different core types may be found.

In this respect molybdenum is probably a typical case and, therefore, the material we will focus on in this paper. Using many-body central-force potentials of the Finnis-Sinclair type [51] constructed for molybdenum [52], the degenerate core structure, shown in Fig. 1a, was found in $[37,38] .{ }^{1}$ The same core structure was also found when using non-central potentials based on a modified generalized pseudopotential theory $[39,40,53,54]$. On the other hand, Fig. 1b shows the core structure obtained in calculations employing the recently developed screened bond-order potentials for molybdenum [43-45]. The most important feature of these potentials, which are based on the tight-binding approach $[42,55,56]$, is that they reflect the most significant quantum mechanical aspect of bonding in transition metals, formation of directional bonds due to the unfilled d-band. Very recently the same non-degenerate core structure in molybdenum was also found in ab initio calculations based on the density functional theory $[46,47,57]$. Thus, at this stage it is not possible to state definitively whether the core of 1/2[1 11 1] screw dislocations in molybdenum is degenerate or non-degenerate. The non-degenerate core structure is most likely the structure found in pure molybdenum since it is predicted from state-of-the-art ab initio calculations. However, it is important to keep in mind that alloying and impurities that are always present in practice may modify the interatomic forces sufficiently for the degenerate core to arise. Indeed, an asymmetric (degenerate) spreading of the screw dislocation core in molybdenum is consistent with recent high-resolution transmission electron microscopy observations [58].

However, it is not the details of the core structure but rather the stress-state dependence of the Peierls stress of the screw dislocations which is of principal importance for physically correct formulation of the flow rules in bcc metals. The strategy for determination of the stress-state dependence of yielding by atomistic simulations is demonstrated
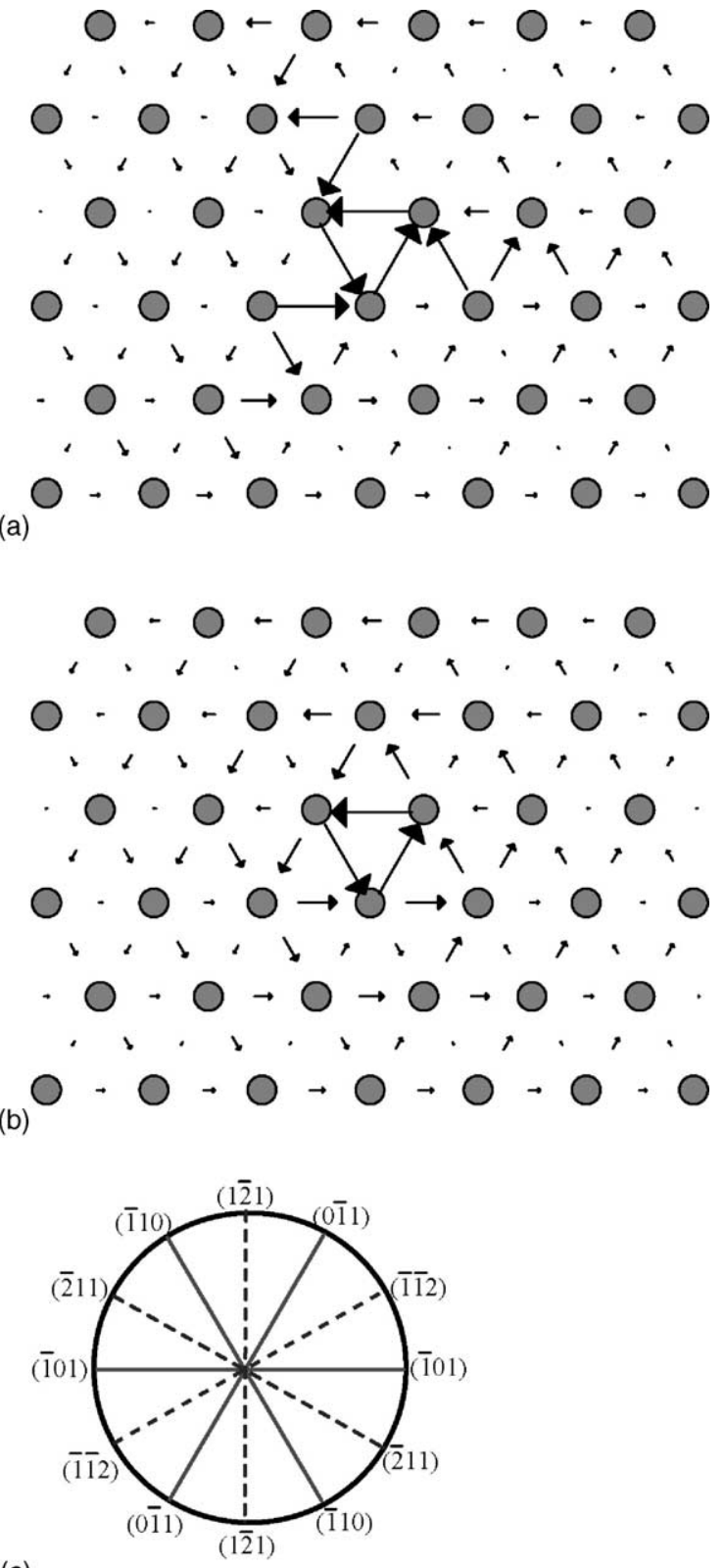

(c)

Fig. 1. Calculated structure of the core of the 1/2[1 11 1] screw dislocation in molybdenum using: (a) central-force many-body potentials [38] and (b) screened bond-order potentials [45]. (c) Orientations of all the $\left\{\begin{array}{lll}1 & 1 & 0\end{array}\right\}$ and

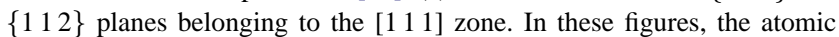
arrangement is shown in the projection perpendicular to the direction of the dislocation line ([ $\left.\left[\begin{array}{lll}1 & 1 & 1\end{array}\right]\right)$. The circles represent atoms within one period without distinguishing that they are positioned in three different layers separated by the distance $a \sqrt{3 / 2}$, where $a$ is the lattice parameter. The pictures are all oriented such that the horizontal rows of atoms correspond to ( 101$)$ planes. The component of the relative displacement parallel to the Burgers vector (screw component) of the neighboring atoms, produced by the dislocation, is depicted as an arrow between them. The length of the arrows is proportional to the magnitude of the displacement normalized by $\mid \mathrm{a} / 6\left[\begin{array}{lll}1 & 1 & 1\end{array}\right]$; the direction of the arrows represents the sign of the displacement.

${ }^{1}$ A non-degenerate core structure was found for this type of potentials for tantalum. 


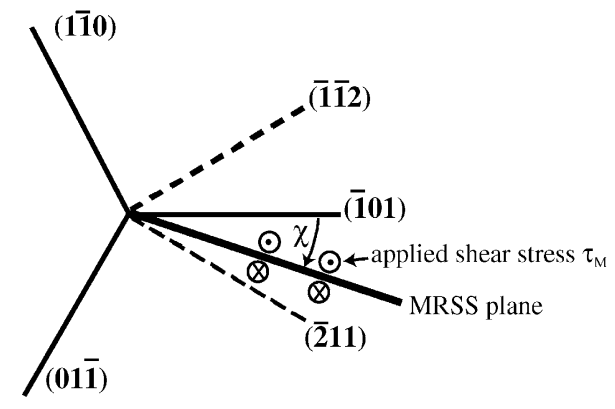

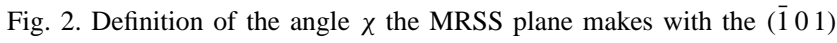
plane.

here for the case of stressing by pure shear in the direction of the Burgers vector. Geometrically, this stressing can be uniquely represented by the angle $\chi$ between the (1 01 ) plane and the maximum resolved shear stress plane, as defined in Fig. 2. For such loading the dislocation in molybdenum moved along the ( $\overline{1} 01)$ plane for all angles $\chi$, but for $\chi$ close to $\pm 30^{\circ}$ when the average glide plane is either $(\overline{2} 11)$ or (1 12$)$. The dependence of the critical resolved shear stress on the MRSSP on the angle $\chi$, calculated by atomistic simulations, is shown in Fig. 3. In this figure, we display results obtained in [38] using central-force many-body potentials, results of recent calculations employing SBOPs [45] and also results of ab initio calculations from Refs. $[46,47]$ and calculations using MGPT potentials from Ref. [41]. It is remarkable that although the core structure is de-

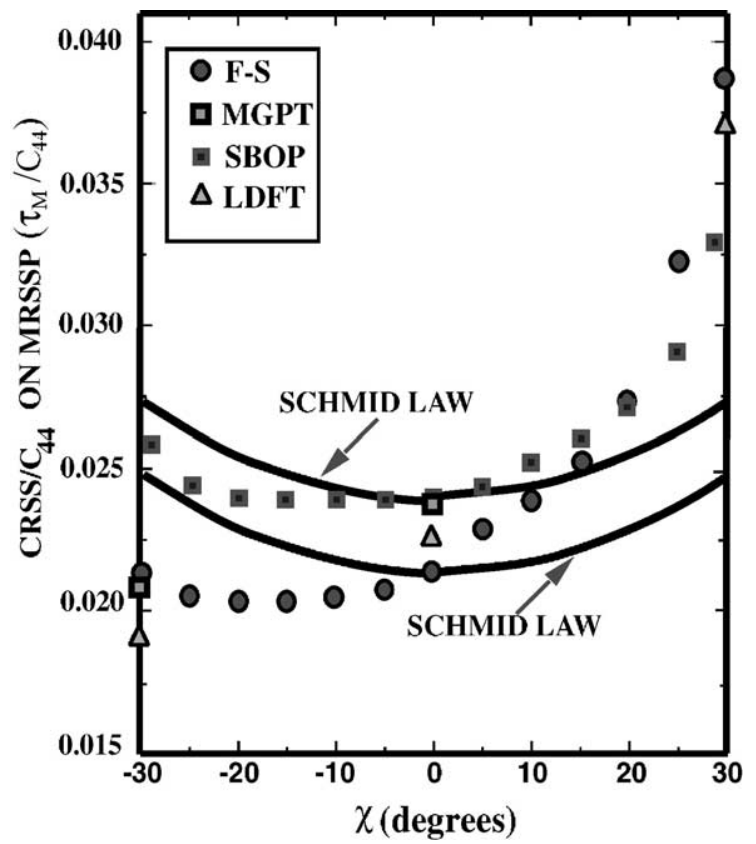

Fig. 3. Dependence of the CRSS on the angle $\chi$ in molybdenum calculated using many-body central-force potentials of the Finnis-Sinclair $(\mathrm{F}-\mathrm{S})$ type [38], screened bond-order potentials (SBOP) [45], MGPT potentials [41] and by an ab initio method based on the LDFT [46,47]. generate in the case of central-force many-body and MGPT potentials and non-degenerate in the case SBOPs and ab initio calculations (see Fig. 1), the MRSSP versus $\chi$ dependencies are similar. The reason is that both types of cores are being altered similarly by the applied stress. This is apparent from Figs. $4 a$ and $b$ that show the core structure of the $1 / 2\left[\begin{array}{lll}1 & 1 & 1\end{array}\right]$ screw dislocation just before it starts to move for central-force many-body potentials and SBOPs, respectively. It is seen that the non-degenerate symmetrical structure transforms into an asymmetrical configuration akin to that of the degenerate configuration.

The results summarized in Fig. 3 clearly demonstrate that the Schmid law, which is also plotted in this figure for the case when the slip plane is the ( 101$)$ plane, does not apply. The reason is that other stress components than the Schmid stress alter the core of the screw dislocation prior to its motion, which is evident from Fig. 4. As discussed in the following section, the stress components that enter the yield criterion representing the results of atomistic studies shown in Fig. 4, are shear stresses on $\left(\begin{array}{lll}\overline{1} & 0 & 1\end{array}\right)$ and $\left(\begin{array}{lll}0 & \overline{1} & 1\end{array}\right)$ planes and both these stresses appear in the yield criterion used in both the continuum modeling of plasticity of both single and polycrystals.

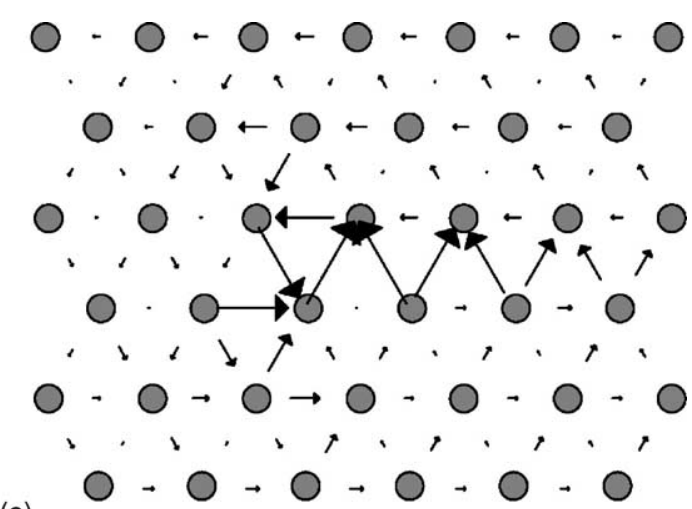

(a)

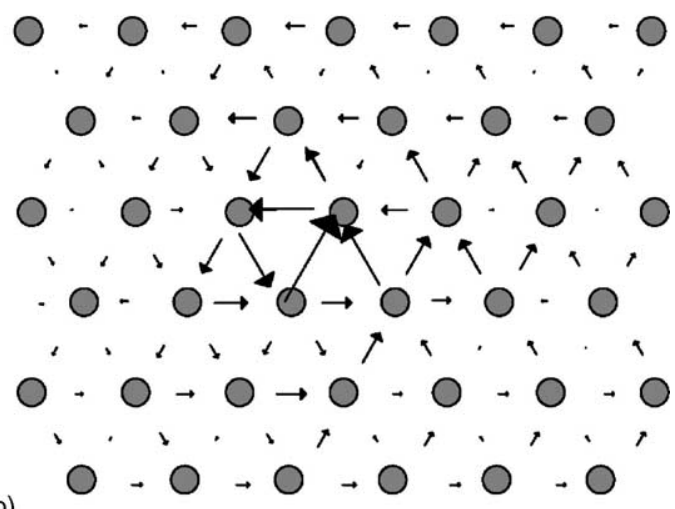

Fig. 4. Core structure of the 1/2[1 11 1] screw dislocation in molybdenum under the effect of the applied shear stress in the $\left(\begin{array}{lll}1 & 0 & 1\end{array}\right)$ plane $\left(\chi=0^{\circ}\right)$. (a) Many-body central-force potentials [38]; (b) screened bond-order potentials [45]. 


\section{Continuum modeling of plastic flow in single crystals of molybdenum}

Continuum models for Schmid-type behavior (fcc and basal slip in hcp) are all based upon the pioneering work of Taylor [59] that has evolved to include capabilities for simulations that account for finite shape change and lattice rotations [1,3,4,29]. Qin and Bassani [30,31] have proposed a framework for non-Schmid behavior and demonstrated how important features of non-planar dislocation cores in $\mathrm{L}_{2}$ intermetallic compounds, such as $\mathrm{Ni}_{3} \mathrm{Al}[17,60]$, can be introduced into a rigorous finite-strain theory. This theory captured the observed orientation dependence and tension-compression asymmetry of the yield stress in the regime of the anomalous increase of the flow stress with temperature. At the same time it suggested significant consequences of non-Schmid behavior for the formation of localized shear bands, which are important in failure mechanisms (for reviews, see $[4,17]$, as well as for polycrystalline response see following section).

Following the suggestion of Qin and Bassani [30,31], the effects of non-glide stresses may enter the flow rules for slip at the single crystal level such that the effective yield stress, $\tau^{* \alpha}$ — which accounts for the effects of non-glide stresses for slip system $\alpha$-is a linear combination of the Schmid stress and other non-glide components of stress tensor:

$\tau^{* \alpha} \equiv \tau^{\alpha}+\sum_{\eta} a_{\eta}^{\alpha} \tau_{\eta}^{\alpha}$

where $\tau^{\alpha}$ is the Schmid stress for the slip system $\alpha, \tau_{\eta}^{\alpha}$ are the non-glide stresses affecting the slip system $\alpha$ and $a_{\eta}^{\alpha}$ are the material parameters that determine the relative importance of the different non-glide stresses (which can depend on the deformation history and temperature); summation is over the total number of non-glide components. The yield criterion for each slip system is then

$\tau^{* \alpha}=\tau_{\mathrm{cr}}^{* \alpha}$

where $\tau_{\mathrm{cr}}^{* \alpha}$ is the yield stress of slip system $\alpha$. If there is no influence of non-glide stresses, this criterion reduces to the Schmid law. In a rate dependent setting, a flow rule can be formulated in the form:

$\dot{\gamma}^{\alpha}=\dot{\gamma}_{0} f\left(\frac{\tau^{\alpha}}{\tau_{\mathrm{cr}}^{* \alpha}-\sum_{\eta} a_{\eta}^{\alpha} \tau_{\eta}^{\alpha}}\right)$

where the function $f$ needs to be determined from the dislocation mobility studies. The atomistic simulations provide the basis for determining the possible slip planes and the important non-glide stress components. Furthermore, from the atomistic simulations of the motion of individual dislocations we directly determine $\tau_{\mathrm{cr}}^{* \alpha}$ and the coefficients $a_{\eta}^{\alpha}$.

Based upon the results of atomistic calculations of the CRSS versus $\chi$ employing central-force potentials for molybdenum [38], shown in Fig. 3 and reproduced in Fig. 5, we have determined that the important non-glide stress

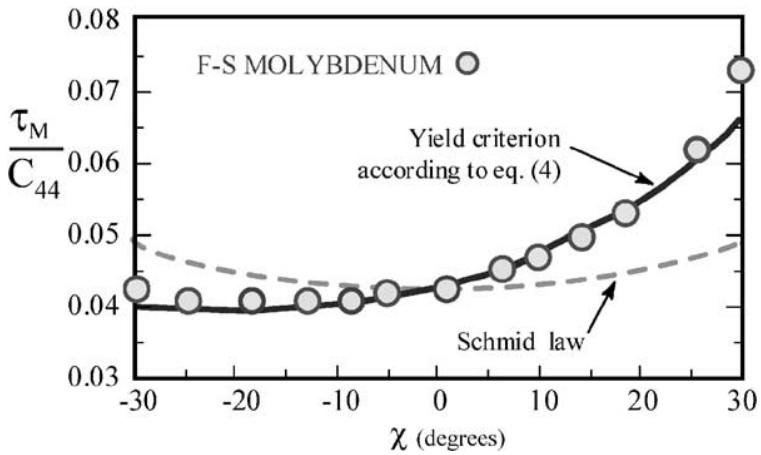

Fig. 5. Dependence of the CRSS on the angle $\chi$ in molybdenum calculated using many-body central-force potentials of the Finnis-Sinclair $(\mathrm{F}-\mathrm{S})$ type [38] and yield criterion given by Eq. (4).

component is the shear stress acting parallel to the Burgers vector in the $(0 \overline{1} 1)$ plane; the slip plane is $(\overline{1} 01)$. The full curve in Fig. 5 is based upon the yield criterion

$\tau^{*}=\tau_{\left(\overline{1}_{01)}\right.}+a \tau_{\left(0 \overline{1}_{1)}\right)}=\tau_{\mathrm{cr}}^{*}$

with $a=0.641$ and $\tau_{\mathrm{cr}}^{*} / C_{44}=0.029$. The corresponding table of crystallographic tensors that suffices for shear parallel to the Burgers vector has been fully determined (and used in the Taylor-type calculation for polycrystals presented below). We note that single crystal flow rules can be readily coupled with the hardening relations in the form of Wu et al. [61] or Cuitiño and Ortiz [62] or the strength relations of Arsenlis and Parks [63].

\section{Polycrystalline response}

In this section, we demonstrate that the effects of nonglide stresses that have their origin at the level of individual dislocations also have significant effect on polycrystalline response. We have very recently formulated a novel quadratic programming problem for non-Schmid behavior at the single crystal level that allows us to perform a Taylor-type calculation for textured polycrystals (an upper bound for non-hardening behavior) - details will be reported elsewhere. Here, we consider a random polycrystal of bec grains with $12\left\{\begin{array}{lll}1 & 1 & 0\end{array}\right\}\left\langle\begin{array}{lll}1 & 1 & 1\rangle\end{array}\right\rangle$ slip systems for which the yielding of each system is defined by an equation of the form of (4), with $\tau_{\mathrm{cr}}^{* \alpha}=\tau_{0}$ taken to be the same on each system. For the case of uniaxial stressing of the polycrystal, the dependence of the macroscopic uniaxial tensile flow stress $\bar{\sigma}$, normalized by the critical hardness of each slip system, is plotted in Fig. 6 as a function of the non-glide-stress parameter $a$. These results show a significant non-Schmid effect at the polycrystal level including a tension-compression asymmetry. Note, that for this material model, the Taylor factor, equal to 3.07 for Schmid type behavior $(a=0)$, decreases nearly linearly with increasing $a$ and is reduced to 2.46 in tension for $a=1$ and a significantly smaller value in compression. Clearly, non-glide stresses also affect the overall 

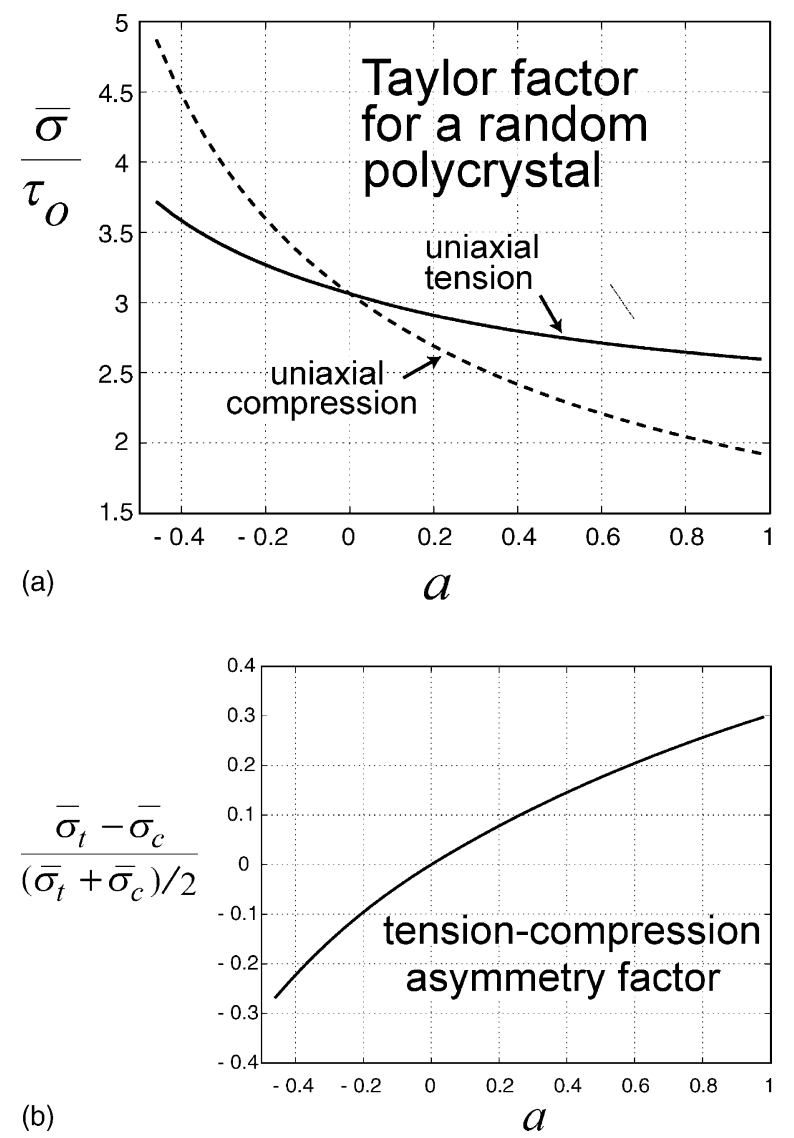

Fig. 6. Macroscopic uniaxial tensile flow stress (a) and its tensioncompression asymmetry (b) for a random bcc polycrystal as a function of the strength of the non-glide stress effect at the slip system level characterized by $a . a=0$ corresponds to the classical Taylor factor of 3.07 times the slip-systems yield stress for Schmid type behavior.

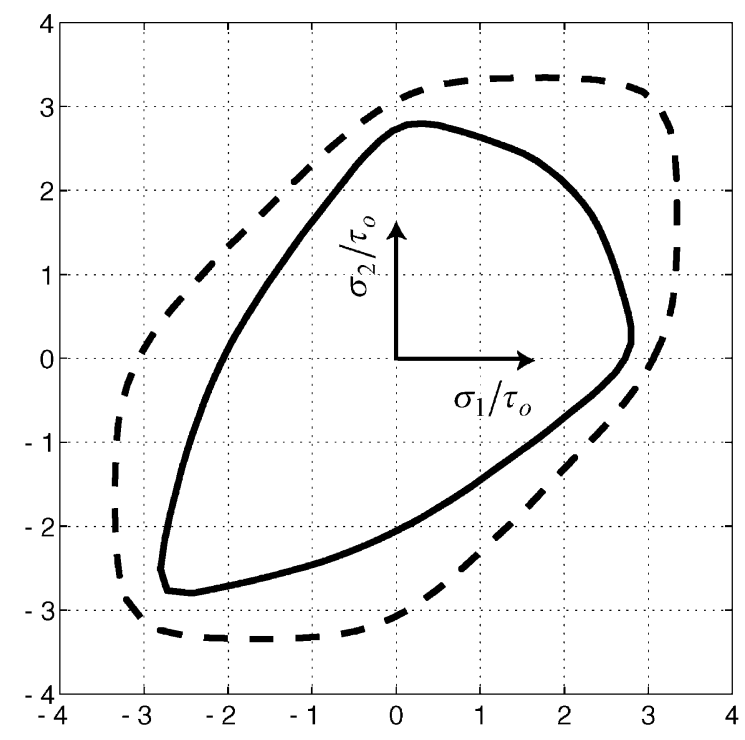

Fig. 7. Two-dimensional plane stress yield surfaces for a random polycrystal: inner locus corresponds to the bcc model with $a=0.6$ and the outer locus to $a=0$ (Schmid behavior). Stresses are normalized by the critical stress on each slip system, $\tau_{0}$. yield surface for polycrystals as shown in plane stress surface presented in Fig. 7. The inner locus is the result for a bcc material with $a=0.6$ (which approximates molybdenum) and outer locus is the Taylor-Bishop-Hill result for Schmid behavior which is approximately the von Mises ellipse. The degree of change in shape of the yield surface is known to have a significant effect on strain localization, limits to ductility and failure. Hence, the effects of non-glide stresses, which have their origin in the dislocation core structures, may, indeed, have significant effects on material behavior at the polycrystalline level.

\section{Conclusions}

We have demonstrated a powerful methodology that builds upon atomistic simulations of dislocation behavior to develop a physically-based continuum theory of crystal plasticity incorporating the effects of non-glide components of the shear stress. This paper has focused on bcc metals, but the phenomenon of stress-state dependence of the Peierls barrier is found in a wide range of materials for which dislocations, most frequently screw dislocations, possess non-planar core structures. The effects of non-glide stresses have been incorporated into a full, three-dimensional continuum theory of multiple slip in bcc crystals. The implications are not only striking at the single crystal level, but significant effects also are predicted at the polycrystalline level. Further studies are underway to explore more general states of stress and other materials.

\section{Acknowledgements}

Discussions with Dr. James Stolken and the assistance of Dr. L. Yin with the polycrystalline calculations are gratefully acknowledged. This research was supported by the NSF Grant no. CMS99-00131 and by the Advanced Strategic Computing Initiative of the US Department of Energy through LLNL, Grant no. B501663.

\section{References}

[1] J.R. Rice, J. Mech. Phys. Sol. 19 (1971) 433.

[2] R. Hill, J.R. Rice, J. Mech. Phys. Sol. 20 (1972) 401.

[3] R.J. Asaro, Adv. Appl. Mech. 23 (1983) 1.

[4] J.L. Bassani, Adv. Appl. Mech. 30 (1994) 191.

[5] E. Schmid, in: Proceedings of the International Congress on the Applied Mechanics, Delft, 1924, p. 342.

[6] J.P. Hirth, J. Lothe, Theory of Dislocations, Wiley/Interscience, New York, 1982

[7] V. Vitek, in: M. Lorretto (Ed.), Dislocations and Properties of Real Materials, The Institute of Metals, London, 1985, p. 30.

[8] M.S. Duesbery, in: F.R.N. Nabarro (Ed.), Dislocations in Solids, vol. 8, Elsevier, Amsterdam, 1989, p. 67.

[9] V. Vitek, Prog. Mater. Sci. 36 (1992) 1.

[10] F. Louchet, J. Phys. IV 3 (1993) 567.

[11] S. Takeuchi, Radiat. Eff. Defect Solids 148 (1999) 333. 
[12] J.W. Christian, in: W.C. Leslie (Ed.), Second International Conference on the Strength of Metals and Alloys, ASM, Metals Park, OH, 1970, p. 31.

[13] J.W. Christian, V. Vitek, Rep. Prog. Phys. 33 (1970) 307.

[14] J.W. Christian, Metall. Trans. A 14 (1983) 1237.

[15] M.S. Duesbery, G.Y. Richardson, CRC Critic. Rev. Solid State Mater. Sci. 17 (1991) 1.

[16] P. Veyssiere, Rev. Phys. Appl. Paris 23 (1988) 431.

[17] V. Vitek, D.P. Pope, J.L. Bassani, in: F.R.N. Nabarro, M.S. Duesbery (Eds.), Dislocations in Solids, vol. 10, Elsevier, Amsterdam, 1996, p. 135.

[18] C. Vailhe, D. Farkas, in: J.A. Horton, I. Baker, S. Hanada, R.D. Noebe, D.S. Schwartz (Eds.), High-Temperature Ordered Intermetallic Alloys VI, vol. 364, Materials Research Society, Pittsburgh, 1999, p. 395.

[19] J. DiPersio, J.C. Doukhan, G. Saada, J. Phys. France 28 (1967) 661.

[20] J. Crampon, J.L. Farvacque, J.C. Doukhan, B. Escaig, Phys. Stat. Sol. (a) 24 (1974) 167.

[21] S. Amelinckx, in: F.R.N. Nabarro (Ed.), Dislocations in Solids, vol. 2, North Holland, Amsterdam, 1979, p. 67.

[22] H. Alexander, in: F.R.N. Nabarro (Ed.), Dislocations in Solids, vol. 7, North-Holland, Amsterdam, 1986, p. 114

[23] R. Jones, Mater. Sci. Eng. B 71 (2000) 24.

[24] J.F. Justo, A. Antonelli, A. Fazzio, Solid State Commun. 118 (2001) 651.

[25] J.-P. Poirier, J. Geophys. Res. 80 (1975) 4059.

[26] J. Dipersio, B. Escaig, Phys. Stat. Sol. (a) 40 (1977) 393.

[27] J.-P. Poirier, B. Vergobbi, Phys. Earth Planetary Interiors 16 (1978) 370.

[28] J.F.W. Bishop, R. Hill, Philos. Mag. 42 (1951) 414.

[29] K.S. Havner, Finite Plastic Deformation of Crystalline Solids, Cambridge University Press, New York, 1992.

[30] Q. Qin, J.L. Bassani, J. Mech. Phys. Sol. 40 (1992) 813.

[31] Q. Qin, J.L. Bassani, J. Mech. Phys. Sol. 40 (1992) 835.

[32] P.B. Hirsch, in: Proceedings of the 5th International Conference on Crystallography, Cambridge University Press, Cambridge, 1960, p. 139.

[33] V. Vitek, Crystal Lattice Defects 5 (1974) 1.

[34] M.S. Duesbery, V. Vitek, J. Cserti, in: C.J. Humphreys (Ed.), Understanding Materials: A Festschrift for Sir Peter Hirsch, The Institute of Materials, London, 2002, p. 165.
[35] M.S. Duesbery, Proc. Roy. Soc. London A 392 (1984) 145.

[36] M.S. Duesbery, Proc. Roy. Soc. London A 392 (1984) 175.

[37] M.S. Duesbery, V. Vitek, Acta Mater. 46 (1998) 1481.

[38] K. Ito, V. Vitek, Philos. Mag. A 81 (2001) 1387.

[39] W. Xu, J.A. Moriarty, Phys. Rev. B 54 (1996) 6941.

[40] W. Xu, J.A. Moriarty, Comp. Mater. Sci. 9 (1998) 348.

[41] S.I. Rao, C. Woodward, Philos. Mag. A 81 (2001) 1317.

[42] A.P. Horsfield, A.M. Bratkovsky, D.G. Pettifor, M. Aoki, Phys. Rev. B 53 (1996) 1656.

[43] D. Nguyen-Manh, D.G. Pettifor, V. Vitek, Phys. Rev. Lett. 85 (2000) 4136.

[44] M. Mrovec, V. Vitek, D. Nguyen-Manh, D.G. Pettifor, L.G. Wang, M. Sob, in: I.M. Robertson, D.H. Lassila, B. Devincre, R. Phillips (Eds.), Multiscale Phenomena in Materials Experiments and Modeling, vol. 578, Materials Research Society, Pittsburgh, 2000, p. 199.

[45] M. Mrovec, Ph.D. Thesis, University of Pennsylvania, Philadelphia, 2002.

[46] C. Woodward, S.I. Rao, Philos. Mag. A 81 (2001) 1305.

[47] C. Woodward, S.I. Rao, Phys. Rev. Lett. 88 (2002) 216402.

[48] V. Vitek, R.C. Perrin, D.K. Bowen, Philos. Mag. A 21 (1970) 1049.

[49] Z.S. Basinski, M.S. Duesbery, R. Taylor, Can. J. Phys. 49 (1971) 2160.

[50] M.S. Duesbery, V. Vitek, D.K. Bowen, Proc. Roy. Soc. London A 332 (1973) 85.

[51] M.W. Finnis, J.E. Sinclair, Philos. Mag. A 50 (1984) 45.

[52] G.J. Ackland, R. Thetford, Philos. Mag. A 56 (1987) 15.

[53] J.A. Moriarty, Phys. Rev. B 42 (1990) 1609.

[54] J.A. Moriarty, in: R.M. Nieminen, M.J. Puska, M.J. Manninen (Eds.), Proceedings in Physics, Many-Atom Interactions in Solids, vol. 48, Springer, Berlin, 1990, p. 158.

[55] M. Aoki, D.G. Pettifor, in: P.M. Oppeneer, J. Kübler (Eds.), Physics of Transition Metals, World Scientific, Singapore, 1993, p. 299.

[56] M. Aoki, D.G. Pettifor, Mater. Sci. Eng. A 176 (1994) 19.

[57] S. IsmailBeigi, T.A. Arias, Phys. Rev. Lett. 84 (2000) 1499.

[58] W. Sigle, Philos. Mag. A 79 (1999) 1009.

[59] G.I. Taylor, J. Inst. Metals 62 (1938) 307.

[60] V. Paidar, D.P. Pope, V. Vitek, Acta Metall. 32 (1984) 435.

[61] T.-Y. Wu, J.L. Bassani, C. Laird, Proc. Roy. Soc. London A 435 (1991) 1.

[62] A.M. Cuitiño, M. Ortiz, Comp. Sim. Mater. Sci. Eng. 1 (1993) 225.

[63] A. Arsenlis, D.M. Parks, Acta Mater. 47 (1999) 1597. 